\title{
Integrability and Non-Integrability of Planar Hamiltonian Systems of Cosmological Origin
}

\author{
Andrzej J MACIEJEWSKI ${ }^{\dagger}$ and Marek SZYDtOWSKI ${ }^{\ddagger}$ \\ $\dagger$ Torun Centre for Astronomy, Nicolaus Copernicus University, Toruń, Poland \\ ¥ Astronomical Observatory, Jagiellonian University, Orla 171, 30-244 Kraków, Poland
}

\begin{abstract}
We study the problem of non-integrability (integrability) of cosmological dynamical systems which are given in the Hamiltonian form with indefinite kinetic energy form $T=\frac{1}{2} g(v, v)$, where $g$ is a two-dimensional pseudo-Riemannian metric with a Lorentzian signature $(+,-)$, and $v \in T_{x} \mathcal{M}$ is a tangent vector at a point $x \in \mathcal{M}$ of the configuration space $\mathcal{M}$.
\end{abstract}

\section{Introduction}

The main aim of this paper is to demonstrate how standard methods of investigation of integrability (non-integrability) work when applied to study systems of cosmological origin. The example of our discussion is a class of two-dimensional Hamiltonian FriedmanRobertson-Walker (FRW) models with scalar fields. The paper contains application of the Ziglin theory and its modification for proving non-integrability of FRW systems.

\section{Direct construction of integrable systems. Integrals linear and quadratic in momenta}

For a system with Hamiltonian

$$
H=\frac{1}{2}\left(p_{x}^{2}-p_{y}^{2}\right)+V(x, y)
$$

let us find all potentials which admit a second integral of motion linear in momenta and having the form

$$
I=A(x, y) p_{x}+B(x, y) p_{y} .
$$

Note that term of zero order in the momenta have been omitted so that $I$ have a good time parity. We have

$$
[I, H]=A_{x} p_{x}^{2}+\left(B_{x}-A_{y}\right) p_{x} p_{y}-B_{y} p_{y}^{2}-\left(A V_{x}+B V_{y}\right)=0,
$$

where subscripts denote partial derivatives and $[\cdot, \cdot]$ denote Poisson brackets.

Since equation (3) must hold identically the following relations are fulfilled $A_{x}=0$, $B_{x}=A_{y}, B_{y}=0, A V_{x}+B V_{y}=0$. The general solution of the above system is $A=\alpha y+\gamma$, $B=\alpha x+\beta$, where $\alpha, \beta, \gamma$ are constants. 
Two cases of different value of $\alpha$ must be considered. First, put $\alpha=0$. Then, the last equation of the above system becomes $\gamma V_{x}+\beta V_{y}=0$ with the solution $V=V(\beta x-\gamma y)$. After rotation in hyperbolic space $(x, i y)$ we have $x \rightarrow X=(\beta x+\gamma y) /\left(\beta^{2}-\gamma^{2}\right)$ and $y \rightarrow Y=(\gamma x+\beta y) /\left(\beta^{2}-\gamma^{2}\right)$; the potential becomes $V=V(X)$ and the corresponding integral is $I=p_{Y}$.

The second case is $\alpha \neq 0$. Without loss of generality we can assume that $\alpha=1$ and then we perform the translation $x \rightarrow x+\beta=X, y \rightarrow y+\gamma=Y$ Equation $A V_{x}+B V_{y}=0$ becomes now $Y V_{X}+X V_{Y}=0$ with the solution $V=V\left(X^{2}-Y^{2}\right)=V(r)$, where $r$ is a radius in two-dimensional Minkowski space with the metric $d s^{2}=d x^{2}-d y^{2}, x=r \cosh \alpha$, $y=r \sinh \alpha$.

The corresponding integral is

$$
I=Y P_{x}+X P_{y}=y \dot{x}-x \dot{y} .
$$

We conclude that the planar potentials which admit a second integral of motion, which is linear in momenta or can be reduced to this form by means of linear point transformations, depend on one variable or are central potentials in the Minkowski space. The second integral of motion is the linear or angular momentum, respectively.

Now, the next step is to search for planar potentials $V(x, y)$ which admit an integral quadratic in momenta

$$
I=A p_{x}^{2}+B p_{x} p_{y}+C p_{y}^{2}+D,
$$

where $A, B, C, D$ are functions of $x$ and $y$. Again the terms, which are linear in momenta, are omitted in order to $I$ possesses a good time parity.

Darboux [2] obtained a general differential equation for a potential of simple classical mechanical system admitting an integral of motion $I$ of the form (5).

From condition $[H, I]=0$ for Hamiltonian (1) and integral (5) we obtain the following equations

$$
\begin{aligned}
& A_{x}=0, \quad B_{x}-A_{y}=0, \quad C_{x}-B_{y}=0, \quad C_{y}=0, \\
& D_{x}=2 A V_{x}+B V_{y}, \quad D_{y}=-B V_{x}-2 C V_{y} .
\end{aligned}
$$

The general solution of $(6)$ is

$$
A=+\alpha y^{2}+\beta y+\gamma, \quad B=+2 \alpha x y+\beta x+\delta y+\epsilon, \quad C=+\alpha x^{2}+\delta x+\xi,
$$

where $\alpha, \beta, \gamma, \delta, \epsilon, \xi$ are constants while integrability conditions on (7) yield the equation

$$
2(A+C) V_{x y}+B\left(V_{x x}+V_{y y}\right)+\left(2 A_{y}+B_{x}\right) V_{x}+\left(B_{y}+2 C_{x}\right) V_{y}=0
$$

where $A, B, C$ are given by (8).

Equation (9) can be treated as a counterpart of classical Darboux equation. It is a necessary and sufficient condition for a potential $V(x, y)$ to admit a second integral of motion which is quadratic in momenta.

\section{Non-integrability of the FRW evolution with scalar fields}

In this section we apply Ziglin theory [6, 7] and its extension [3] for proving non-integrability of FRW dynamical system. This approach is attractive because Ziglin's theory in 
its general formulation is applicable for Hamiltonian systems with a Hamiltonian function having an arbitrary form. However, for an effective application of this theory one needs to determine the monodromy group of (normal) variational equations associated with a particular solution. This can be done only for very special cases, and because of it, several effective formulations of non-integrability theorems where formulated for systems with prescribed form of the Hamiltonian (e.g. [4, 5]). In fact these special formulations consider natural systems with constant positive definite and diagonal form of the kinetic energy.

\subsection{Outline of Ziglin theory}

The fundamental papers of Ziglin [6, 7] gave the formulation of a very basic theorem about non-integrability of analytic Hamiltonian systems. The idea of Ziglin approach lies in a deep connection between properties of solutions on a complex time plane and the existence of first integral. This idea takes its origins in works of S W Kovalevskaya and A M Liapunov.

Here we formulate only the facts needed to state the relevant theorems in fairly simple settings. We consider $\mathbf{C}^{2 n}$ as a complex symplectic manifold with the canonical symplectic structure $\Omega$. A Hamiltonian vector field $v_{H}$ is determined by a complex Hamiltonian function $H: \mathbf{C}^{2 n} \longrightarrow \mathbf{C}$ by relation $\Omega\left(v_{H}, \cdot\right)=d H$. We assume that Hamilton's analytic equations

$$
\frac{d}{d t} z=v_{H}(z), \quad z=\left(z_{1}, \ldots, z_{2 n}\right) \in \mathbf{C}^{2 n}, \quad t \in \mathbf{C},
$$

have a non-equilibrium solution $z=\varphi(t)$. To simplify the exposition we assume that this solution lies in a two dimensional invariant plane

$$
\Pi=\left\{\left(z_{1}, \ldots, z_{2 n}\right) \in \mathbf{C}^{2 n} \mid z_{i}=0, i=1, \ldots, 2(n-1)\right\} .
$$

The phase curve $\Gamma=\left\{\varphi(t) \in \mathbf{C}^{2 n} \mid t \in \mathbf{C}\right\}$ is a Riemannian surface with a local coordinate $t$. Together with equations (10) we consider also variational equations along solution $\varphi(t)$

$$
\frac{d}{d t} \xi=A(t) \xi, \quad A(t)=\frac{\partial v_{H}}{\partial z}(\varphi(t)) .
$$

This system separates into the normal and the tangential subsystems. In our settings this separation takes a very simple form, as the matrix $A(t)$ has a block diagonal structure. We consider the normal variational equations (NVE)

$$
\frac{d}{d t} \eta=B(t) \eta, \quad \eta \in \mathbf{C}^{2(n-1)}
$$

where $B(t)$ is the $2(n-1) \times 2(n-1)$ upper diagonal block of matrix $A(t)$. We choose a point $t_{0} \in \mathbf{C}$ and a matrix of fundamental solutions of NVE $X(t)$, defined in a neighborhood of $t_{0}$. With a close path $\alpha$ on complex time plane starting and ending at point $t_{0}$ we can associate a matrix $S \in \mathrm{GL}(2(n-1), \mathbf{C})$ in the following way. We integrate along the path $\alpha$ NVE (12), i.e., we make an analytic continuation of $X(t)$ along this path. As a result from the fundamental solution $X(t)$ we obtain another fundamental solution $Y(t)$. From the general theory of linear systems it follows that $Y(t)=S X(t)$ for some $S \in \mathrm{GL}(2(n-1), \mathbf{C})$. Because our system is Hamiltonian, $S$ is a symplectic matrix, i.e, $S \in \operatorname{Sp}(2(n-1)$, C). 
In this way, considering all possible paths, we obtain a matrix representation of the first homotopy group $\pi_{1}(\Gamma)$ of $\Gamma$. It forms a finitely generated subgroup of $\operatorname{Sp}(2(n-1), \mathbf{C})$ and it is called the monodromy group. We denote it $M$.

Let us take an element of monodromy group $g \in M$. Its spectrum has the form

$$
\operatorname{spectr}(g)=\left(\lambda_{1}, \lambda_{1}^{-1}, \ldots, \lambda_{n-1}, \lambda_{n-1}^{-1}\right), \quad \lambda_{i} \in \mathbf{C} .
$$

The element $g$ is called resonant if

$$
\prod_{l=1}^{n-1} \lambda_{l}^{k_{l}}=1 \quad \text { for some } \quad\left(k_{1}, \ldots, k_{n-1}\right) \in \mathbf{Z}^{n-1} \backslash\{0\} .
$$

Theorem 1 [Ziglin, 1982]. Let us assume that there exists a non-resonant element $g \in$ $M$ and moreover that in a connected neighbourood of $\Gamma$ the Hamiltonian system possesses $n-1$ meromorphic first integrals which are functionally independent on $H$. Under these hypothesis, if ge $=\lambda e$ for $\lambda \in \mathbf{C}$ and $e \in \mathbf{C}^{2(n-1)}$, then for any element $g^{\prime} \in M$ it holds $g\left(g^{\prime} e\right)=\lambda^{\prime}\left(g^{\prime} e\right)$ for some $\lambda^{\prime} \in \mathbf{C}$.

In the case of a system with two degrees of freedom this theorem can be formulated in a more operational way

Theorem 2. Let us assume that there exists a non-resonant element $g \in M$. If there exists some other element $g^{\prime} \in M$ such that

1) $\operatorname{Tr} g^{\prime} \neq 0$ and $g g^{\prime} \neq g^{\prime} g$, or

2) $\operatorname{Tr} g^{\prime}=0$ and $g g^{\prime} g \neq g^{\prime}$,

then there is no additional meromorphic first integral functionally independent on $H$ in a connected neighborhood of $\Gamma$.

The main difficulty with the application of Ziglin theorem is the determination of the monodromy group of NVE. Only in very special cases we can do this analytically. Yoshida $[4,5]$ developed Ziglin approach for special cases when the Hamiltonian of a system has a natural form and the potential is a homogeneous function. In this case we can find a particular solution in the form of 'straight line solution' and the normal variational equations for it can be transformed to a product of certain copies of hyper-geometric equations for which the monodromy group is known. This allows to formulate adequate theorems in algorithmic form. Below we describe it for an Hamiltonian system with two degrees of freedom.

Consider the Hamiltonian

$$
H=\frac{1}{2}\left(p_{1}^{2}+p_{2}^{2}\right)+V\left(q_{1}, q_{2}\right), \quad\left(q_{1}, q_{2}, p_{1}, p_{2}\right) \in \mathbf{C}^{4},
$$

where $V\left(q_{1}, q_{2}\right)$ is a homogeneous function of degree $k$, i.e. $V\left(C q_{1}, C q_{2}\right)=C^{k} V\left(q_{1}, q_{2}\right)$. In the generic case this system has straight line solutions of the form $q_{1}=C_{1} \phi(t), q_{2}=C_{2} \phi(t)$, where $\phi(t)$ solves the nonlinear equation $\ddot{\phi}=-\phi^{k-1}$ and $\left(C_{1}, C_{2}\right) \neq(0,0)$ are solutions of the following system $C_{1}=\partial_{1} V\left(C_{1}, C_{2}\right), C_{2}=\partial_{2} V\left(C_{1}, C_{2}\right)$. The variational equations take the form

$$
\left[\begin{array}{c}
\ddot{\xi} \\
\ddot{\eta}
\end{array}\right]=-\left[\begin{array}{ll}
V_{11} & V_{12} \\
V_{21} & V_{22}
\end{array}\right]\left[\begin{array}{l}
\xi \\
\eta
\end{array}\right](\phi(t))^{k-2},
$$


where $V_{i j}=\partial_{i} \partial_{j} V\left(C_{1}, C_{2}\right)$ for $i, j=1,2$. Since the Hessian of $V$ is symmetric it is diagonalizable by an orthogonal transformation and the system separates to

$$
\begin{aligned}
& \ddot{\xi}=-\lambda_{1} \phi^{k-2}(t) \xi, \\
& \ddot{\eta}=-\lambda_{2} \phi^{k-2}(t) \eta,
\end{aligned}
$$

where $\lambda_{1}, \lambda_{2}$ are the real eigenvalues of the Hessian. Let us note that it is not true for indefinite systems where the Hessian is not a symmetric matrix.

It can be shown that the Hessian of $V$ at $C=\left(C_{1}, C_{2}\right)$ has an eigenvalue $\lambda_{1}=k-1$. Thus, its second eigenvalue is equal $\lambda:=\lambda_{2}=\operatorname{tr} V\left(C_{1}, C_{2}\right)-(k-1)$, and it is called the integrability index. Equation (15) is the normal variational equation. It can be transformed to the hyper-geometric equation. The monodromy matrices of this equation are parametrized by $\lambda$ and the conditions of Ziglin's theorem put restrictions on the values of $\lambda$ : we can identify those values of $\lambda$ for which our system is not integrable (more precisely: does not possess an additional meromorphic first integral). To state it accurately let us define

$$
I_{k}(p)=\left[\frac{k}{2} p(p+1)-p, \frac{k}{2} p(p+1)+p+1\right], \quad p \in \mathbf{N},
$$

and

$$
N_{k}=\mathbf{R} \backslash \bigcup_{p \in \mathbf{N}} I_{k}(p) .
$$

Then it follows that the Hamiltonian system (13) with a homogeneous potential of degree $k$ is not integrable if the integrability index $\lambda$ corresponding to a certain straight line solution belongs to $N_{k}$.

\subsection{Application to the FRW Hamiltonian system}

Let us consider the flat Friedman-Robertson-Walker system with self-interacting field [1]. After the canonical transformation $\left(p_{1}, q_{1}\right) \rightarrow\left(i p_{1},-i q_{1}\right)$ the Hamiltonian function has the form

$$
H=\frac{1}{2}\left(p_{1}^{2}+p_{2}^{2}\right)+V\left(q_{1}, q_{2}\right), \quad V\left(q_{1}, q_{2}\right)=\frac{\Lambda}{4} q_{1}^{4}-\frac{\mu}{2} q_{1}^{2} q_{2}^{2}+\frac{\lambda}{2} q_{2}^{4},
$$

where $(\Lambda, \lambda, \mu) \in \mathbf{R}^{4}$ are parameters of the problem. The equation $q=V^{\prime}(q), q=\left(q_{1}, q_{2}\right)$ has the following solutions

$$
z_{1}=\left( \pm \lambda^{-1 / 2}, 0\right), \quad z_{2}=\left(0, \pm \lambda^{-1 / 2}\right), \quad z_{3}=\left( \pm \sqrt{\frac{\lambda+\mu}{\Lambda \lambda-\mu^{2}}}, \pm \sqrt{\frac{\lambda+\mu}{\Lambda \lambda-\mu^{2}}}\right) .
$$

The integrability indices for these points are

$$
\lambda_{i}=-\operatorname{tr} V^{\prime \prime}\left(z_{i}\right)-3, \quad i=1,2,3
$$

and

$$
\lambda_{1}=-\frac{\mu}{\Lambda}, \quad \lambda_{2}=-\frac{\mu}{\lambda}, \quad \lambda_{3}=\frac{\lambda_{1} \lambda_{2}-2\left(\lambda_{1}+\lambda_{2}\right)+3}{1-\lambda_{1} \lambda_{2}} .
$$

Thus, from the Yoshida criterion it follows that if there is $l \in\{1,2,3\}$ such that $\lambda_{l} \in N_{4}$ then the FRW system has no additional meromorphic first integral that is functionally independent with $H$. 


\subsection{Application of the Morales-Ramis theory}

Recent results of Morales-Ruiz and Ramis (see [3]), extend the Ziglin theory by connecting it with the differential Galois theory. Results obtained till now are important from a theoretical point of view and moreover give very strong tools to study applied problems.

In this approach we restrict ourselves to prove the lack of complete Liouville integrability but instead of using the monodromy group we investigate the differential Galois group of variational equations. Usually Galois group is bigger than the monodromy group and because of this one can achieve non-integrability results more easily. Lack of space does not allow us to present the basic idea of this interesting and very effective approach. Here we mention only the results that one obtains by applying this theory to a case considered by Yoshida, i.e. a natural Hamiltonian system with a homogeneous potential of degree $k$. For such a situation Morales-Ruiz and Ramis proved the following. Let $\lambda_{i}$ be an eigenvalue (different than $k-1$ ) of the Hessian of the potential evaluated at a point which corresponds to a straight line solution and let $k$ be the degree of homogeneity of the potential. Then, if the system is integrable (in the Liouville sense) then $\left(\lambda_{i}, k\right)$ (for all straight line solutions) belong to a certain fully described discrete set. For details see Theorem 5.1 in [3]. This gives a stronger result than the Yoshida criterion because the Yoshida criterion implies that under the same assumptions $\lambda_{i}$ belongs to set $\bigcup_{p \in \mathbf{N}} I_{k}(p)$,
but this set has a non-empty interior.

Application of the theorem cited above to the FRW Hamiltonian system gives the following result. Let us introduce the following three discrete sets

$$
\begin{aligned}
& I_{1}=\{p(2 p-1) \mid p \in \mathbf{Z}\}, \\
& I_{2}=\left\{\frac{1}{8}\left[-1+16\left(\frac{1}{3}+p\right)^{2}\right] \mid p \in \mathbf{Z}\right\}, \\
& I_{3}=\left\{\frac{1}{2}\left[\frac{3}{4}+4 p(p-1)\right] \mid p \in \mathbf{Z}\right\} .
\end{aligned}
$$

Then if $\left\{\lambda_{1}, \lambda_{2}, \lambda_{3}\right\} \not \subset I=I_{1} \cup I_{2} \cup I_{3}$ the system is non-integrable. Here $\left\{\lambda_{1}, \lambda_{2}, \lambda_{3}\right\}$ are given by (19).

It is interesting to select those cases when $\left\{\lambda_{1}, \lambda_{2}, \lambda_{3}\right\} \subset I$, i.e. those values of parameters for which system can be integrable. Note that $\lambda_{3}$ is a symmetric function of $\lambda_{1}$ and $\lambda_{2}$, implying that it is enough to know $\left(\lambda_{1}, \lambda_{2}\right)$. It is easy to observe that if $\lambda_{1}=1$ or $\lambda_{2}=1$ then $\lambda_{3}=1$. Assume for example that $\lambda_{2}=\lambda_{3}=1$. Then $\mu=-\lambda$ and $\lambda_{1}=\lambda / \Lambda$. If the system is integrable then 1 and $\lambda / \Lambda \subset I$.

\section{Acknowledgements}

The paper was supported by the KBN grant no. 2 P03D 01417. 


\section{References}

[1] Calzetta E and Hasi C E, Class. Quantum Grav., 1993, V.10, 1825.

[2] Darboux G, Arch. Neerl., 1901, V.6, N 11, 371.

[3] Morales-Ruiz J J, Differential Galois Theory and Non-Integrability of Hamiltonian Systems, Bürkhauser, New York, 1998.

[4] Yoshida H, Phys. D, 1986, V.21, N 1, 163-170.

[5] Yoshida H, Phys. D, 1987, V.29, N 1-2, 128-142.

[6] Ziglin S L, Funktsional. Anal. i Prilozhen., 1982, V.16, N 3, 30-41.

[7] Ziglin S L, Funktsional. Anal. i Prilozhen., 1983, V.17, N 1, 8-23. 